\title{
Discussion to paper of Mr McGregor and Mr Buchan
}

\section{Chairman: DR Dollfus}

Mr MCGREgor. First of all before commencing I would like to acknowledge the fact that whilst Mr Buchan and myself are plastic surgeons working in the Regional Centre for the Lothian region in Scotland most of our patients that we describe in this paper were patients of the Spinal Injuries Unit, Edenhall Hospital, Musselburgh.

DR FRANKel. Mr Chairman I'd like to clarify a point because the name of Bruce Bailey is used and as his address is the same as that of the National Spinal Injuries Centre I'd like to make it quite clear he is not part of that Centre. We don't use flaps at all.

Mr NusEibeh (G.B.). Well I think I have to disagree with you because the principle of treating pressure sores was completly ignored there. The paraplegic always is exposed all the time and if that is so then the minimum disturbance of soft tissue is required must be put high in consideration and I must say it's been completely ignored, and one of the causes of pressure sores is the presence of one prominence. What have you done about it?

MR MCGREGOR. I did in fact say, if you listened, that I removed the bony prominence with the technique of Guttmann making a pseudo-tumour attack into the pressure sore. I did say that and we do remove any bone prominence.

Mr NUSEIBEH. Well that is an extravagant way of treating a pressure sore when you can excise and close up pressure sores quite easily without any flaps.

Mr MCGREGOR. Well again I think if you were listening I did infer that we believed that for trochanteric sores this flap is useful but we are certainly less happy than the United States people are in the closure of ischial sores. So we are partly in agreement but I would like to see whether conservative measures in the long term produce any better results. What if the sores are extremely large, you will not get them to close directly.

Mr NUSEIBEH. Simple excision and simple closure of that ischial sore would have been ten times easier without that extravagant flap.

MR MCGREGor. With due respect Sir again if you had been listening, I stated at the very beginning, that while we would not necessarily do that now, it was to demonstrate the technique.

MR NUSEIBEH. If that is so you should show that with your indications.

MR MCGREGor. Trochanteric pressure sores that are too big to be closed directly.

MR NUSEIBEH. Well I'm afraid that in a paper of mine given to the Society at Stoke Mandeville, we have revealed something like over 250 cases of pressure sores, I've forgotten the exact number of trochanteric sores, and we never did a single flap on them. We are not doing measures we got the facts plus vital conclusions.

Mr MCGREGOR. Can I ask you how long the patients stay in hospital?

Chairman. This is becoming I think a matter to be discussed between you two. I think we've discussed about this practically at every meeting and at every meeting we have a heated discussion so I think it's hot enough for everybody to go back home to refresh themselves. 